\title{
Immersive Virtual Reality Education Application - Four Development Iterations along Design Science Research Methodology
}

\author{
Jani Holopainen \\ University of Jyväskylä \\ jamaholo@jyu.fi
}

\author{
Antti Lähtevänoja \\ University of Jyväskylä \\ anjulaht@student.jyu.fi
}

\author{
Tuure Tuunanen \\ University of Jyväskylä \\ tuure@tuunanen.fi
}

\begin{abstract}
This study introduces a research and development process of an immersive Virtual Reality (VR) education application. Altogether four application development iterations are showcased along the Design Science Research methodology. The results show how initial problems change and new problems occur during the course of a long-term DSR project with multiple iterations. Moreover, the study results confirm various previous findings, for example, that VR works better on higher learning levels such as application-level and personalization is a way to achieve this. In addition, 360 photos and videos were found to be easy and costefficient ways to increase personalization of $V R$ applications. The results also suggest complementary and parallel use of online courses and VR applications in order to enable scalability. We also found positive learning experiences, interactions, and teacher image to be achieved with VR especially among small tech-savvy group of students. However, personalized learning paths and positive learning results are the preconditions for upbeat learning experiences.
\end{abstract}

\section{Introduction}

Education and training have become one of the major application areas for the immersive Virtual Reality (VR). Immersive VR can be defined as a digital environment with high sense of presence and flow of tasks consumed with Head-Mounted-Displays (HMDs) [36]. However, the most studies in the field are providing only specific, limited and less mature knowledge about educational VR applications [28]. Studies providing this kind of knowledge usually focuses on exploring the possibilities of VR education, for example, by investigating what are the most suitable application fields for VR technology (e.g., [2, 9 14]). These studies are focusing on users' motivations, engagement, and learning outcomes of various VR applications. However, as a literature review presented in this paper reveals, these studies are not considering in detail the problem-solutions fields, e.g., constructs, methods, models, design principles or technological rules [13]. As a result, the field lacks more generalizable design models and theories to guide the practice and future research.

One thing that seems to be completely missing from the research field is long-term design studies with multiple development iterations [28], that is, building design theories [13]. According to Gregor and Hevner [13], only long-term or multi-case design studies can produce enough knowledge about the artifact problem and solution fields that can be called a design theory. These findings lay down the motivation for this study at hand. The purpose of this study thus is to present what are the problems and solutions identified during a longterm development process of an immersive VR education application? With these findings we are proposing more mature design knowledge and theories in the field of immersive VR education applications.

Following DSR methodology [35], this study presents an iterative development of an immersive VR education platform applicable in various educational fields and disciplines. Most importantly the study introduces initial, emerging and changing problems during the development process. Solving these problems set the main motivations for the inclusion of the research. In addition, the study shows accumulating design knowledge guiding the development of subsequent solution artifacts. We believe that these findings are useful and generalizable to both practitioners and scholars developing and studying immersive VR education applications.

\section{Methodology}

Peffers et al. [35] suggest that a methodology in DSR include three elements: conceptual principles, practical rules, and process definition. In the following, we describe how we consider these elements in our study.

In terms of the main principles for DSR we adopt the problem relevancy [20] theory ingrained solution artifacts [15] interpretation of design process as a 
research outcome [33] and, finally, seeking improvements instead of the absolute truth [39].

What it comes to the practical rules, we consider Gregor and Hevner [13] who suggest consideration of multiple cases and/or long-term research in order generate new and incremental design knowledge. In addition, vom Brocke et al. [44] suggests detailed descriptions of contexts, objectives, artifacts, and design processes in order to generate more knowledge about the problem and solution spaces. Furthermore, they suggest that the design knowledge (i.e., kernel and design theories as well as knowledge about solution artifacts, design processes and artifact evolution) is accumulated during the research and development projects over time. Lukyanenko et al. [27] adds that consideration of multiple artifacts and the detailed description and elaboration of the design process reveals issues about the validity and reliability of the design knowledge contributions.

Finally, DSR methodology [35] offers a process model to conduct DSR research. They introduce an iterative process constituting of six steps: problem identification, solution objectives, developSment, demonstration, evaluation, and communication. It should be noted that as suggested in the seminal work by Peffers et al. [35] as well as in many latter methodological notes (e.g., [35]), there is no single and strict way to conduct DSR, but the DSR methodology rather offers an overall framework and mental model to present the findings.

In addition to addressing the main principles and the practical rules, in the results section we offer a detailed description of the DSR process including multiple evaluations conducted with various methods including experiments, scales, open-ended questions and observations.

\section{Literature on immersive VR education applications}

This section introduces previous research from the field of immersive VR education applications. Following the main principles for DSR introduced in the previous methodology section $([15,30,33,39])$, we are especially interested in investigating the problem field, suggested solutions, design processes as well as design knowledge contributions by the existing research.

A study by Agrawal et al. [2] identifies a problem where young drivers are at a great risk of being involved in car crashes as they have inexperience in hazard anticipation skills. In their suggested solution a VRbased training is established, where the data from the driver's performance is captured and the performance can be played back. They validate the VR design by comparing VR-training to other hazard mitigation programs to see whether the hazard anticipation training can be enhanced while maintaining the mitigation training effect. They found that VR-based hazard anticipation training can greatly improve the performance of untrained novice drivers. Their study confirms with many others suggesting that VR works in training simulations which are otherwise dangerous or impossible to arrange (e.g., [29, 40]).

Another study by Herrero et al. [14] states that for children with ASD, it is sometimes hard to simulate social situations for training emotional and social skills. Their solution was a VR-based training, where social situations are recreated in an immersive setting. Their sought validity in their results by comparing VR and conventional trainings as a control group. The results suggest that VR works in training social situations and skills. Similar results are suggested by also some other VR studies (e.g., [18, 21].

Freitas et al. [9] found that abstract subjects in computer science (e.g., memory management) are sometimes hard to teach. To solve that problem their tested VR-based simulator where students can learn how a computer manages its memory. They included immersive and interactive elements to their solutions suggesting that these are the main factors when seeking generalizability of the results. Comparisons between VR and desktop virtual environment showed that VR works well in teaching abstract subjects such as memory management and allocation in computer science. Similar results are achieved by Lui et al. [26] in science education.

As these above studies suggest the learning subject and context matters in the case of VR education applications. As a result, VR seems to work best in training simulations which are otherwise dangerous or impossible to arrange, training social situations and skills as well as teaching abstract subjects. In addition, immersive and interactive VR environments have been found to enhance student motivation, engagement, experience, and contextual learning ([17, 31]. VR is also showcased to enhance deep learning or higher levels of learning e.g., application, analysis, evaluation, and creation of things [5, 6, 34]. Finally, 360 photos and videos as part of the VR can produce personalized environments and contents [16].

What seems to be complete missing from the existing research field is long-term design and development studies also recognized in Lähtevänoja et al. [28]. Only long-term or multi-case design studies [13] and detailed descriptions of contexts, objectives, artifacts, and design processes [44] can produce generalizable and valid design knowledge contributions [27]. Therefore, the objective for this study is to present what are the problems and solutions identified during a 
long-term development process of an immersive VR education application.

These existing VR studies form the theoretical foundation and existing design knowledge base for the problem identification and suggested solutions under each application development iteration. In addition to those, there are several other studies guiding the development which are introduced more in detail in next sections.

\section{Results}

The results introduce altogether four application development iterations taking place between 2016-2021 and their main design knowledge contributions for VR learning environments. Each iteration description (1-4) and their paragraphs follow DSR methodology and its phases including problem identification, solution objectives, development, demonstration, evaluation and communication [35].

\subsection{Iteration 1 - Interactive content and scalability}

The development of the first solution artifact started with the requirement and need to offer a nationwide online course targeted for high school students where Massive Online Courses (MOOCs) were seen as a scalable option [1, 22]. The solution artifact was also aimed to provide information about the forest sector and so to attract students to apply to the corresponding fields at the university. The identified problem with the online courses was related to the student motivation, engagement, experience (e.g., [4, 10, 12, 38]) and contextual learning (e.g., [11]).

The solution artifact included a MOOC with interactive 360-environments. These 360-environments were made available for desktop and VR cardboards which we sent around the country of Finland (altogether 500 pieces). The inclusion of VR cardboards was based on the suggestions from the previous research that immersive and interactive VR environments can enhance student motivation, engagement, experience, and contextual learning $[17,31]$ and therefore coping the identified problems areas.

The artifact development began with filming the 360-environments. Contents for the MOOC -course were collected by showing the 360-environments for the experts with carboards and interviewing them how that particular environment related to their work or research and what we should teach student about these topics. Interviews were transcribed, edited, and organized on a Moodle-based MOOC with multiple choice questions and short essay assignments. After that mockup of the interactive 360-environments were drafted to be used with desktop computers. The desktop-version the 360environment could be explored by dragging with a mouse and hotspots were opened with a click. In a VRversion the surrounding was explored just turning the head and hotspots were opened after staring them few seconds while a countdown clock (a circle filling up)

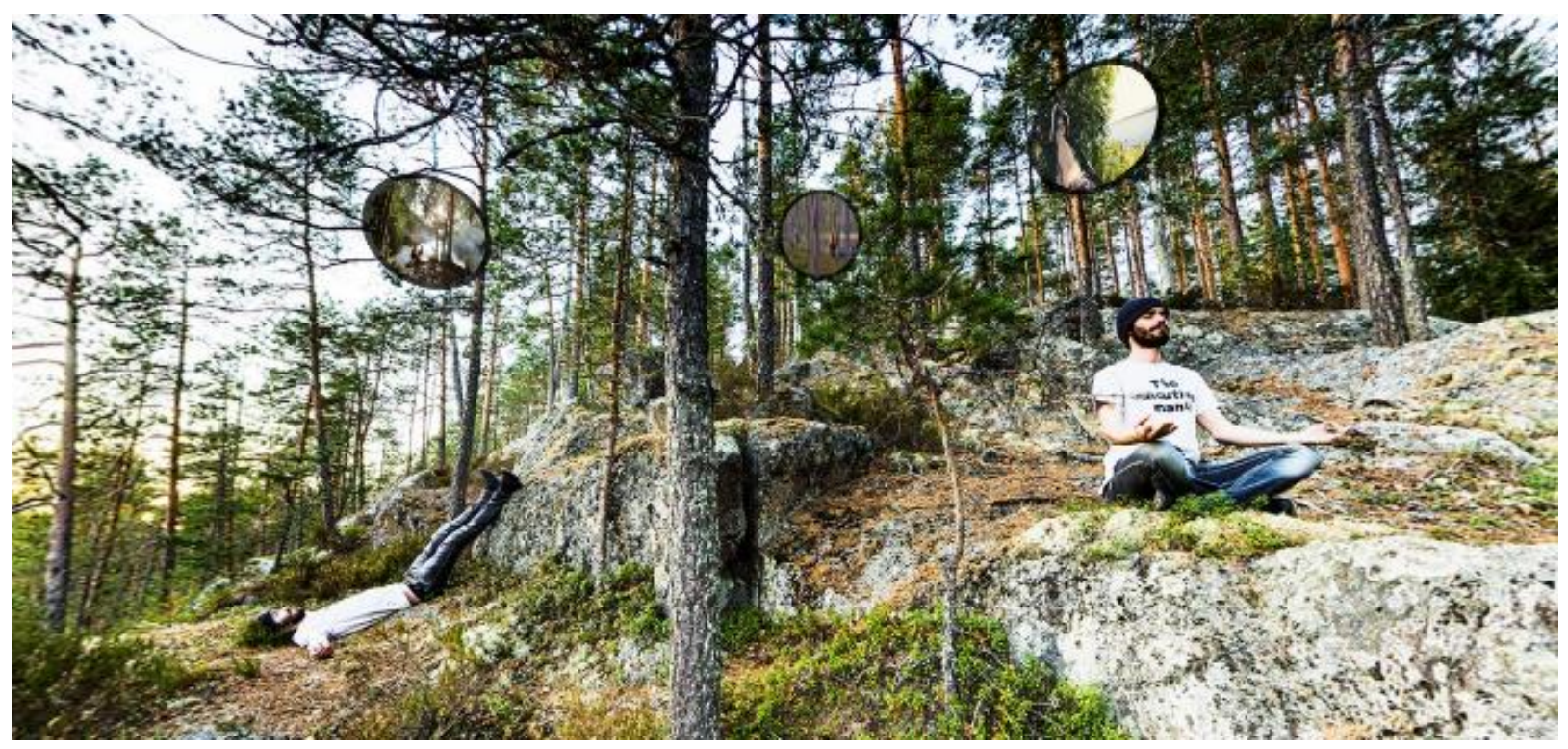

Figure 1. A screen capture of the 360 desktop-environment with interactive hotpots providing information boxes and/or voice narratives (same contents available for the $360-V R$ environment consumed with cardboards). 
was shown when the hotspot was about to be opened. Both versions were identical in terms of the content and interactions - only the device was different. Under the hotspots there was info boxes and/or voice narratives presented for the users (Figure 1).

The demonstration took place in three high schools nationwide and altogether 107 students were surveyed and interviewed in an experiment, where students were divided into three groups to whom the same course content was showcased only with different presentation modes: 1) text and photos, 2) text and 360 desktopenvironment, and 3) text and 360 VR-environment.

The evaluation of the between-subjects experiment showed that the learning results (remembering and understanding) were best among the students who participated the second group i.e., 360 desktopenvironment. This also implies that some sort of interactivity has positive effect on learning. However, there was no difference between the groups in terms of motivation, engagement, and experience. Instead, there was a small tech-savvy group of students who favored 360 and VR technologies, however, the group of students against them was even larger.

The results offered several implications that were also aligned with some previous studies. First, in terms of the learning results, VR seemed to underperform with the lower levels of learning such as remembering and understanding. Instead, as showcased also by some previous research, VR learning environments outperform many other learning environments on the higher levels of learning e.g., application, analysis, evaluation, and creation [5, 6, 34]. Secondly, our results were contradictory compared to some previous results showing positive correlations between VR use and some indirect learning effects such as motivation, engagement, and experience (e.g., [17]). As a result, we concluded that the learning outcome may also mediate these indirect learning effects i.e., if students do not perceive to learn, either the learning motivation, engagement and experience cannot be positive. Finally, the finding of small tech-savvy group of students preferring new technologies indicated the need for providing different learning environments and paths for the students, i.e., adaptive learning materials [43]. All in all, the iteration 1 showed that scalability is achievable with MOOCs but also with VR.

\subsection{Iteration 2 - Personalization and higher levels of learning}

The second artifact iteration was set to overcome the main problem of low learning results achieved from the first development round. The main solution objectives included to create higher level learning tasks i.e., application, analysis, evaluation and/or creation. To do so, we chose to use personalization features as there was some evidence from the research that with personalization higher levels of learning could be achieved [45]. Adding personalization features also required more advanced Head Mounted Display (HMD) VR technology with hand-controllers, i.e., HTC Vive. This decision was done at the cost of scalability as HTC Vive HMDs were much more costly compared to cardboards and they required more powerful computers as well as personal tutoring to get started with the use.

The development of artifact was conducted using Unreal Engine -game engine [42]. Personalization features included choice of surrounding, 3D model imports, drawings, and markings, recoding and viewing recordings and an avatar figure. The application also enabled two different user interfaces: teachers and students. Student interface was otherwise the same as the teachers, but the recording-feature was disabled.

For the demonstration, a teacher specialized in crafts prepared weaving teaching recordings. The recordings were showcased for 97 university level students. The demonstration was a between-subjects experiment with three different teaching recording: teaching in VR, 3D video and $2 \mathrm{D}$ video (Figure 2 ).

In the evaluation -part, the starting level, perceived affordances, and learning were tested with surveys and assignments. The test for the learning was an assignment to draw right weaving composition which can be considered as an application -level learning task. The evaluation results showed that the group assigned to the learning in VR outperformed in terms of learning: $75 \%$ of the VR group completed the assignment correctly, followed by $68 \%$ in the $3 \mathrm{D}$ group and $50 \%$ in the $2 \mathrm{D}$ group. In addition, the evaluation results suggested that personalized learning, multi-sensory effects, immersion, interactivity, 3D-dimensionality, easy-ofuse as well as motivation towards the content and technology were perceived significantly higher for VR compared to two other environments.

The same technology artifact was also demonstrated in three other learning contexts: machinery, biology, and spatial mathematics. For each of these contexts both VR and 3D video learning environments were prepared and 


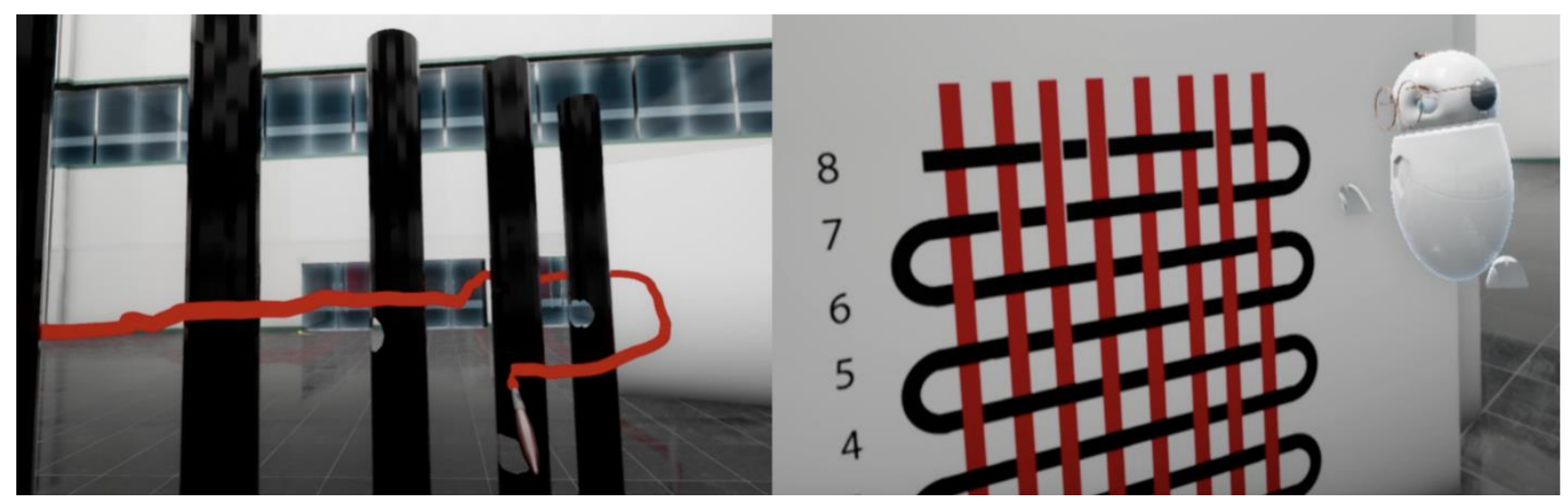

Figure 2. Screenshots from 3D (left) and 2D -videos (right), while the VR recording was exactly the same as the 3D video but consumed with the HMD and so allowing free move and change of viewpoint.

altogether 100 students participated the betweensubjects experiment. Unlike in the first evaluation, this evaluation tested again lower levels of understanding (remembering and understanding) just like the evaluation during the iteration 1 . The results were also exactly the same - VR underperformed compared to the other learning environment. The experiment was also measuring teachers' competence and goodwill which seemed to transmit better in VR compared to the 3D video application, while in terms of teachers' credibility there was no difference.

These findings confirm that the VR outperforms some other learning environments only on the application -learning level of Bloom's Taxonomy. The assumption also remains that it might outperform against some other learning environments also on other higher learning levels such as in analysis, evaluation, and creation, but there is no research evidence on that yet. In addition, the learning level seemed to be the only significant factor for the success of VR: choosing different learning contexts did not seem to affect the result. The hypothesis created during the iteration 1 was confirmed that motivation and many other experience measurements were significantly more positive for VR when also the learning results were positive. Personalization was proved to be a good way to enable higher level learning environments for VR and thus improving the learning results.

\subsection{Iteration $3-360$ photo and video environments and advanced pedagogical models}

The iteration 2 was followed by introductions and discussions with the university teachers in various disciplines potentially adopting the VR teaching and learning application. The money was not an issue as the application and the technical support were provided free of charge. However, the teachers were reluctant in adopting the VR application on their courses. The issues were related to the effort and time required to incorporate new technologies and methods to their course syllabuses. They also felt that although the online 3D model libraries were full of various contents, those were not satisfactory to their own teaching purposes. In multiple discussions emerged the idea of incorporating 360 photos and videos to create personalized environments and contents fast and cheaply for VR, which was also supported by previous research [16]. In addition, the solution objective for the iteration 3 was to further evaluate the higher levels of learning by incorporating various higher level pedagogical models for teaching and learning [24].

In addition to the features introduced in the previous iteration, the iteration 3 development started with adding a new technology feature named 360-sphere. This feature includes 360 photos or videos from a real world and a play-area in the middle of it allowing free movability and interactions (Figure 3).

In terms of the developed and demonstrated teaching models, the VR course included introduction lecture about the learning objectives, contents and methods, self-exploration of teacher-recorded VR content (example outcome), group discussions and designs to create own VR content, roleplay (recording content for VR), analysis of self-recorded content, communicating self-recorded content in VR. As a result, the student assignment outcome was a recording made in VR with an analysis and narrative on the course content (negotiations situation and related theories). This recording was made available to be viewed in VR or on a desktop video, where the student avatar was giving the analysis and narrative on the course content (Figure 3).

The developed and demonstrated VR course artifact was compared with a classical example of Moodlebased online course with another course context 
("Copyright of meme and image"). This online course consisted of conventional Massive Open Online Course (MOOC) elements such as open access, only few assignment deadlines, text, figures, tables, videos, links to extra material, multiple choice questions and two assignments on discussion forums. All in all, the online course can be described as a classical all-round instantiation of a Moodle -course design and related theories (e.g., [8, 32]).

In terms of the evaluation results, the comparison of the VR course artifact (5 student participants) and the online course (11 student participants) was made based on the Cognitive Affordances of Technologies framework (CAT) incorporating various pedagogical models [7]. Online questionnaires were sent to the student participants after their completion of the course. The results indicate that the VR course works well in experimental learning, discursive learning, and supportive learning while these are the weaknesses for the online environment. Learning by doing is supported in both environments. The strength of the online course is that it supports critical thinking, conceptual change, and self-regulated learning, while these are the weak points of the Mixed Reality environment. By implication, the results suggest that the course designs should consider both VR and online course elements in order to provide a broad spectrum of different learning outcomes.

\subsection{Iteration 4 - Simple content production and consumption with adapted pedagogical model}

The previous iteration and VR artifact turned out to be burdensome for both teachers and students. Only a half-day demonstration event required lots of preparations and planning from the teacher who also needed a technical assistant to run the demo course. The multiple teaching methods and contents were well organized during the half-day demo course, so the students were not confused, but it was obvious that the risk for confusion and anxiety increases with full-length courses. In addition, the scalability requirement presented in the first iteration was completely lost in the latter iterations.

These identified problems initiated the next iteration round with solution objectives being all the previously achieved solutions including scalability $[1,22]$ interactive content [17, 31] higher level of learning through personalization [45] 360 photo and video environments [16] as well as advanced pedagogical models [24]. In addition to those, we added simple content production for the teachers and simple consumption for the students as solutions objectives. Finally, a new pedagogical model was needed to be adapted to all other solution objectives and accumulated design knowledge (see e.g., [23]).

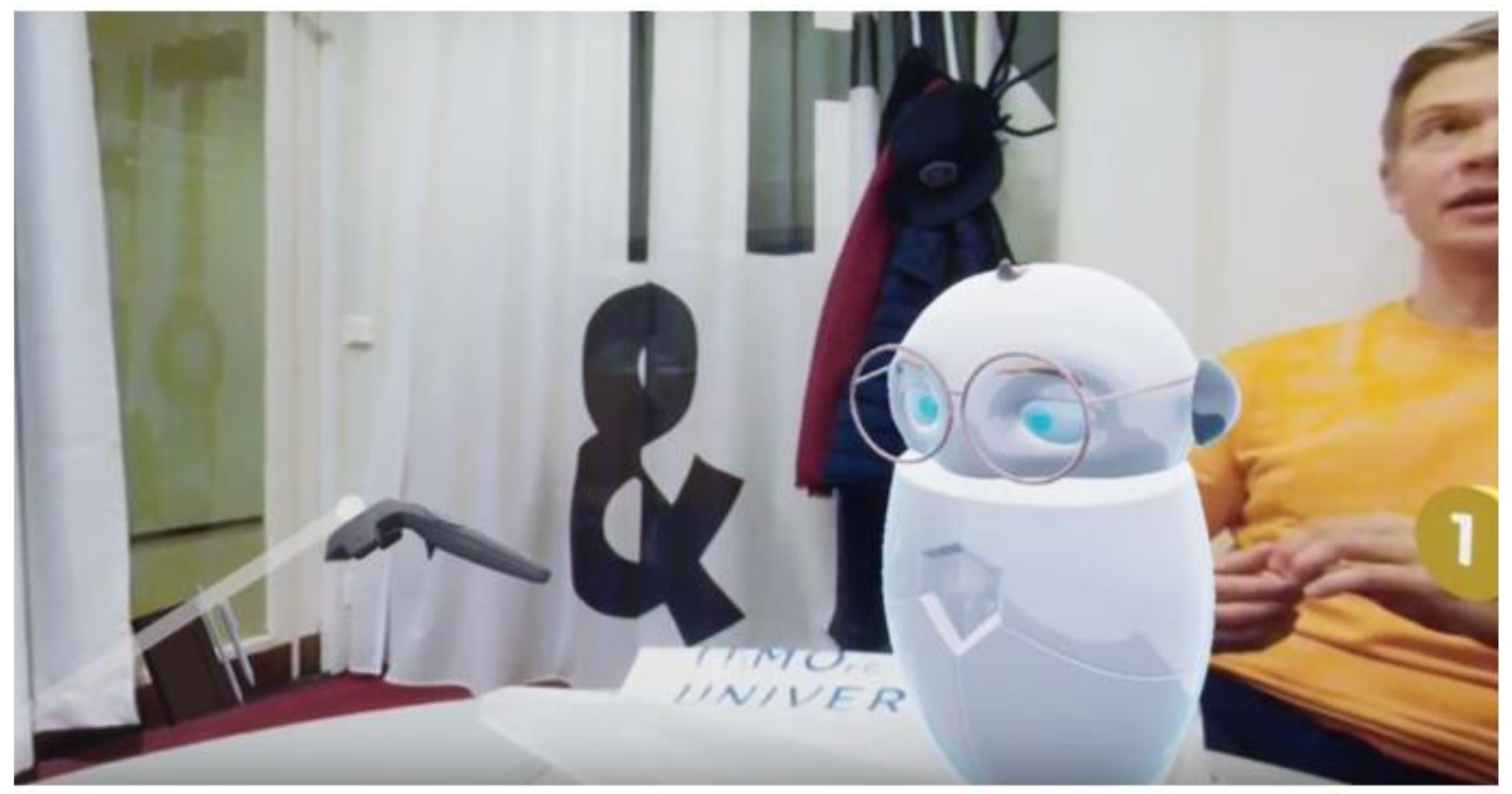

Figure 3. 360-sphere with a play-area in the middle allowing free movability and interactions for the user (an avatar figure). 
As a result, we started to design and develop an artifact combining online and VR course elements. The online -part concentrated on teaching theories, while in the VR -part students would apply the theory into the real surroundings. For the VR -part the teachers' workload was minimized: all they needed to do is to make and download a 360 photo or video from the course context (e.g., a negotiation situation). Another possibility is to search and download 3D environment or model e.g., from the online library. After creating and downloading a course context environment on an online terminal, teacher prepares and downloads subjects related the course context (e.g., theories, models, factors, elements, components, notes etc.). These subjects can be in various formats such text, hyperlinks, figures, tables, 3D models etc. These subjects are downloaded in random order in the online terminal. Now the VR assignment for the students is ready. Students can consume the VR content with standalone Oculus HMDs or from their own desktops / mobile devices making the solution scalable. Students' task is to browse the environment and select and place subjects

Table 1. The summary of the results in terms of identified problems, suggested solutions, design process and design knowledge contributions during four research and development -rounds.

\begin{tabular}{|c|c|c|c|c|}
\hline Iteration \# & $\begin{array}{l}\text { Identified problems } \\
\text { from the field and } \\
\text { literature }\end{array}$ & $\begin{array}{l}\text { Theory ingrained } \\
\text { suggested solutions }\end{array}$ & $\begin{array}{l}\text { Design process } \\
\text { description }\end{array}$ & Design knowledge contributions \\
\hline 1 & $\begin{array}{l}\text {-How to provide } \\
\text { nationwide course? } \\
\text {-Low learning } \\
\text { experiences and } \\
\text { contextual learning } \\
\text { with online courses } \\
\text { (e.g., Barak et al. } \\
\text { 2016; Gašević et al. } \\
\text { 2014; Giesbers et al. } \\
\text { 2013; Shapiro et al. } \\
\text { 2017; Giani 2004) }\end{array}$ & $\begin{array}{l}\text {-Scalable MOOCs and } \\
\text { VR cardboards } \\
\text { (Kizilcec et al. 2013; } \\
\text { Admiraal et al. 2015) } \\
\text {-Interactive contents } \\
\text { including } 360 \text { and VR } \\
\text { environments with } \\
\text { hotspots ensuring } \\
\text { motivation, } \\
\text { engagement and } \\
\text { contextual learning } \\
\text { (Huang et al. 2019; } \\
\text { Mystakidis et al. 2014) }\end{array}$ & $\begin{array}{l}\text {-MOOCs and VR } \\
\text { cardboards chosen } \\
\text { following the existing } \\
\text { studies promising } \\
\text { interactivity and } \\
\text { scalability, however, no } \\
\text { other contemplations } \\
\text { made }\end{array}$ & $\begin{array}{l}\text {-Scalability possible with MOOCs and } \\
\text { VR cardboards } \\
\text {-Some evidence that interactivity } \\
\text { improves learning } \\
\text {-Some evidence that VR does not } \\
\text { work on lower learning levels } \\
\text {-Small tech-savyy group favoring } 360 \\
\text { and VR techs }\end{array}$ \\
\hline 2 & $\begin{array}{l}\text {-Low learning results } \\
\text { with VR }\end{array}$ & $\begin{array}{l}\text {-Creation of higher } \\
\text { level learning tasks } \\
\text { e.g. application etc. } \\
\text { with personalization } \\
\text { (Yoshinov and lliev } \\
\text { 2018) }\end{array}$ & $\begin{array}{l}\text {-No other options than } \\
\text { personalization } \\
\text { considered to create } \\
\text { higher level learning } \\
\text {-Several learning } \\
\text { contexts considered but } \\
\text { not in all learning levels }\end{array}$ & $\begin{array}{l}\text {-Some evidence that VR works on } \\
\text { application -learning level. } \\
\text {-Learning context seems not to have } \\
\text { significant effect on learning in VR (on } \\
\text { lower learning levels) } \\
\text {-VR can enhance positive image on } \\
\text { teacher } \\
\text {-Learning outcome mediate learning } \\
\text { experiences } \\
\text {-Personalization is one way to } \\
\text { generate higher level learning }\end{array}$ \\
\hline 3 & $\begin{array}{l}\text {-Lack of suitable 3D } \\
\text { models and } \\
\text { environments } \\
\text {-Lack of } \\
\text { understanding about } \\
\text { higher level learning } \\
\text { and VR }\end{array}$ & $\begin{array}{l}\text {-360 photo and video } \\
\text { environments (Hu et } \\
\text { al. 2017) } \\
\text {-Creating advanced } \\
\text { pedagogical models to } \\
\text { test higher levels of } \\
\text { learning (Korhonen et } \\
\text { al. 2019) }\end{array}$ & $\begin{array}{l}\text {-Various pedagogical } \\
\text { models included and } \\
\text { considered but it's hard } \\
\text { to cover them all } \\
\text {-VR course artifact } \\
\text { compared to well } \\
\text { presentative online } \\
\text { course, but course } \\
\text { contexts were different }\end{array}$ & $\begin{array}{l}\text { - } 360 \text { tech appeared to be fast, cheap } \\
\text { and personalized way to create VR } \\
\text { content } \\
\text {-Some evidence that VR and online } \\
\text { courses are complementary, and } \\
\text { their parallel use is advised }\end{array}$ \\
\hline 4 & $\begin{array}{l}\text {-With several } \\
\text { advanced } \\
\text { pedagogical models } \\
\text { for VR courses } \\
\text { teachers' efforts too } \\
\text { big and risk of anxiety } \\
\text { for students } \\
\text {-Lack of scalability in } \\
\text { previous solutions }\end{array}$ & $\begin{array}{l}\text {-Those presented } \\
\text { above plus: } \\
\text {-Simple model for } \\
\text { teachers to generate } \\
\text { VR content on an } \\
\text { online terminal } \\
\text {-Simple model for } \\
\text { students to carry out } \\
\text { VR assignments } \\
\text {-A new adapted } \\
\text { pedagogical model } \\
\text { (Komlevand } \\
\text { Dneprovskaya 2018) }\end{array}$ & $\begin{array}{l}\text {-Scalability of both } \\
\text { models for students and } \\
\text { teachers considered } \\
\text {-Scalability ensured with } \\
\text { the choice of tech } \\
\text { (standalone Oculus) } \\
\text {-Different learning } \\
\text { ensured by combining } \\
\text { online and VR elements }\end{array}$ & \\
\hline
\end{tabular}


from the listing prepared by the teacher. The subject placements should be done on right place and on right time (if video) in the environment. The placements should follow the theories taught on the online part of the course. These kinds of tasks for the students are envisaged to consider at least contextual learning, experimental learning, supportive learning and learning by doing elements.

Further, in this iteration version there is no other analytics about the students' correct answers than a student report or multiple-choice assignment on the course online page to indicate which subjects were placed, where they were placed and on which time. In this regard, teachers are encouraged to add extra / unsuitable subjects to test the students. In the future iterations also automatic and artificial intelligence based analytics are possible to automatize the feedback and grading for the students.

This iteration is at its development stage, so no further demonstrations or evaluations are available yet.

\subsection{Summary of results}

The summary (Table 1) of the results is organized according to the adopted methodology. The summary shows the shifting problems and solutions during the course of a long-term DSR project with multiple iterations. The summary also shows the efforts made during the design processes and how all this contribute to the evolving design knowledge. In the description of the design process and following design knowledge contributions we consider criteria such as amount of cases / study contexts, time span, level of details in descriptions of contexts, objectives, artifacts, and processes, which contribute to the novelty, validity and reliability of the design knowledge contributions (e.g., $[13,27,44])$.

The summary of the results is discussed in more detail in the next section.

\section{Discussion}

The results show how initial problems change and new problems occur during the course of a long-term DSR project with multiple development iterations. This finding is in line with e.g., [13, 27, 44] highlighting the importance of multi-perspective, multi-case and/or long-term DSR in order generate novel, valid and reliable design knowledge. However, as shown in our literature review, these kinds of studies have been completely missing in the field of immersive VR education application research. As a result, we suggest that the results of this long-term DSR study with multiple iterations provide more mature design knowledge to the field and some nascent design theory proposals.

Aligned with the previous research (e.g., [5, 6, 34, $27,44]$ ) also our results confirm that VR outperforms other video learning environments at the application learning level. This is confirmed with several studies with multiple cases and perspectives over-time, so we propose this finding as a nascent design theory. Notion of nascent is added, as we believe there are still many learning contexts without any research. Considering more and various learning contexts the future research could specify which learning contexts confirm with this proposal. In addition, there is lack of research considering other higher learning levels, e.g., analysis, evaluation, and creation, which should be adopted in the future research. Our results suggest that personalization is a good way to reach the higher learning levels, but the future research could consider also other means. There are also different types of personalization (e.g., [41]) which should be considered. One type that we considered was incorporating 360 photos and videos to VR which appeared to be fast, cheap, and personalized way to create VR content.

The aforementioned notion of studying more different learning contexts should be considered despite of our finding that learning context seems not to have significant effect on learning in VR. However, this finding was tested only on lower learning levels (understanding and remembering) and with only three learning contexts, so further research in this regard is required especially testing deep and higher levels of learning. It should be noted that majority of the existing VR literature suggests that the learning context matters (e.g., $[2,9,14])$ but this research vein is not considering the different learning levels.

Another finding was that VR seems to be possible to be provided in scalable way especially with parallel MOOCs. In addition, VR and MOOCs seemed to be complementary to each other in terms of the learning outcomes. These are some new findings as previous research has not paid any attention how to scale VR or to combine it with some other methods or technologies. Therefore, the future research should contemplate also other methods and technologies as well as their scalability and learning outcomes to test and establish alternative pedagogical models.

Our results confirm that with VR it is possible to achieve interactions and further positive learning experiences and results $[17,31]$. In addition, we were able to find out that the learning outcome mediates learning experiences i.e., if students do not perceive to learn, either the learning motivation, engagement and experience cannot be positive. Furthermore, VR seemed 
to enhance the positive image on teacher, which is a new finding to the field.

Finally, the finding of small tech-savvy group of students preferring new technologies indicated that there is a need to provide different learning environments and paths for the students and VR is capable to fulfill that need.

\section{Conclusions}

This study made various findings contributing to the existing literature and design knowledge base in the field of VR education applications. We are confident that these findings will offer some guidance also for other VR development projects and designs. However, this study considered only MOOCs and VR applications and proposed the best possible solutions from this perspective which is also a limitation. As a result, for the existing and emerging problems also some other platforms or technologies could provide equally good or even better solutions which should be sought by the future research. In addition, for the future research our study showed that with a long-term DSR project and multiple application development iterations more detailed problem definitions, solution artifacts as well as design processes could be introduced all contributing to more valid design knowledge in the field.

\section{References}

[1] Admiraal, Wilfried, Bart Huisman, and Olga Pilli. "Assessment in massive open online courses." Electronic Journal of E-learning 13.4 (2015): pp. 207-216.

[2] Agrawal, Ravi, Michael Knodler, Donald L. Fisher, and Siby Samuel. "Virtual reality headset training: Can it be used to improve young drivers' latent hazard anticipation and mitigation skills." Transportation research record 2672, no. 33 (2018): 20-30.

[3] Barak, Miri, Abeer Watted, and Hossam Haick. "Motivation to learn in massive open online courses: Examining aspects of language and social engagement." Computers \& Education 94 (2016): pp. 49-60.

[4] Barak, Miri, Abeer Watted, and Hossam Haick. "Motivation to learn in massive open online courses: Examining aspects of language and social engagement." Computers \& Education 94 (2016): pp. 49-60.

[5] Bertrand, Jeffrey, Ayush Bhargava, Kapil Chalil Madathil, Anand Gramopadhye, and Sabarish V. Babu. "The effects of presentation method and simulation fidelity on psychomotor education in a bimanual metrology training simulation." In 2017 IEEE Symposium on 3D User Interfaces (3DUI), pp. 59-68. IEEE, 2017.

[6] Bhargava, Ayush, Jeffrey W. Bertrand, Anand K. Gramopadhye, Kapil C. Madathil, and Sabarish V. Babu. "Evaluating multiple levels of an interaction fidelity continuum on performance and learning in near-field training simulations." IEEE transactions on visualization and computer graphics 24, no. 4 (2018): 1418-1427.

[7] Dabbagh, Nada, and Susan Dass. "CATS: A tool for identifying the cognitive affordances of learning technologies." thannual (2013): 401.

[8] Elias, T. (2010). Universal instructional design principles for Moodle. The International Review of Research in Open and Distributed Learning, 11(2), 110-124.

[9] Freitas, Luiz Felipe Santos, Alex Sandro Rodrigues Ancioto, Rita de Fátima Rodrigues Guimarães, Valéria Farinazzo Martins, Diego Roberto Colombo Dias, and Marcelo de Paiva Guimarães. "A Virtual Reality Simulator to Assist in Memory Management Lectures." In International Conference on Computational Science and Its Applications, pp. 810-825. Springer, Cham, 2020.

[10] Gašević, Dragan, Vitomir Kovanović, Srećko Joksimović, and George Siemens. "Where is research on massive open online courses headed? A data analysis of the MOOC Research Initiative." International Review of Research in Open and Distributed Learning 15, no. 5 (2014): 134-176.

[11] Giani, Umberto. "3.5. Evolutionary Epistemology and Dynamical Virtual Learning Networks." Global Health Informatics Education 109 (2004): 182.

[12] Giesbers, Bas, Bart Rienties, Dirk Tempelaar, and Wim Gijselaers. "Investigating the relations between motivation, tool use, participation, and performance in an e-learning course using web-videoconferencing." Computers in Human Behavior 29, no. 1 (2013): 285-292.

[13] Gregor, Shirley, and Alan R. Hevner. "Positioning and presenting design science research for maximum impact." MIS quarterly (2013): pp. 337-355.

[14] Herrero, Jorge Fernández, and Gonzalo Lorenzo. "An immersive virtual reality educational intervention on people with autism spectrum disorders (ASD) for the development of communication skills and problem solving." Education and Information Technologies 25, no. 3 (2020): 1689-1722.

[15] Hevner, Alan R., Salvatore T. March, Jinsoo Park, and Sudha Ram. "Design science in information systems research." MIS quarterly (2004): 75-105.

[16] Hu, Hou-Ning, Yen-Chen Lin, Ming-Yu Liu, Hsien-Tzu Cheng, Yung-Ju Chang, and Min Sun. "Deep 360 pilot: Learning a deep agent for piloting through 360 sports videos." In 2017 IEEE Conference on Computer Vision and Pattern Recognition (CVPR), pp. 1396-1405. IEEE, 2017.

[17] Huang, Yu-Chih, Sheila J. Backman, Kenneth F. Backman, Francis A. McGuire, and DeWayne Moore. "An investigation of motivation and experience in virtual learning environments: a self-determination theory." Education and Information Technologies 24, no. 1 (2019): 591-611.

[18] Holopainen, Jani, Osmo Mattila, Petri Parvinen, Essi Pöyry, and Tuure Tuunanen. "Sociability in Virtual Reality: Evaluations of Three Iterative Application Versions along a Design Science Research Process." ACM Transactions on Social Computing 4, no. 1 (2021): 1-21.

[19] Hyett, Nerida, Amanda Kenny, and Virginia DicksonSwift. "Methodology or method? A critical review of 
qualitative case study reports." International journal of qualitative studies on health and well-being 9.1 (2014)

[20] Hrastinski, Stefan. "The potential of synchronous communication to enhance participation in online discussions: A case study of two e-learning courses." Information \& Management 45.7 (2008): pp. 499-506.

[21] Kahlon, Smiti, Philip Lindner, and Tine Nordgreen. "Virtual reality exposure therapy for adolescents with fear of public speaking: a non-randomized feasibility and pilot study." Child and adolescent psychiatry and mental health 13.1 (2019): 47.

[22] Kizilcec, René F., Chris Piech, and Emily Schneider. "Deconstructing disengagement: analyzing learner subpopulations in massive open online courses." Proceedings of the third international conference on learning analytics and knowledge. (2013)

[23] Komleva, Nina, and Natalia Dneprovskaya. "Technologies for creating and acquiring knowledge in the open information environment." Technologies for the European Conference on E-Learning (ECEL). (2018)

[24] Korhonen, A-M., Sanna Ruhalahti, and Marjaana Veermans. "The online learning process and scaffolding in student teachers' personal learning environments." Education and Information Technologies 24, no. 1 (2019): 755-779.

[25] Liu, Dejian, Kaushal Kumar Bhagat, Yuan Gao, TingWen Chang, and Ronghuai Huang. "The potentials and trends of virtual reality in education." In Virtual, augmented, and mixed realities in education, pp. 105-130. Springer, Singapore, 2017.

[26] Lui, Michelle, Rhonda McEwen, and Martha Mullally. "Immersive virtual reality for supporting complex scientific knowledge: Augmenting our understanding with physiological monitoring." British Journal of Educational Technology 51.6 (2020): pp. 2181-2199.

[27] Lukyanenko, Roman, Jeffrey Parsons, and Binny Samuel. "Artifact sampling: using multiple information technology artifacts to increase research rigor." Proceedings of the 51st Hawaii International Conference on System Sciences. (2018)

[28] Lähtevänoja, Antti, Jani Holopainen, Mikko Vesisenaho, and Päivi Häkkinen. "Developing design knowledge and a conceptual model for virtual reality learning environments." In Designing, Deploying, and Evaluating Virtual and Augmented Reality in Education, pp. 100-123. IGI Global, 2021.

[29] Dede, Chris, Tina A. Grotzer, Amy Kamarainen, and Shari J. Metcalf. "Virtual reality as an immersive medium for authentic simulations." In Virtual, augmented, and mixed realities in education, pp. 133156. Springer, Singapore, 2017.

[30] March, Salvatore T., and Gerald F. Smith. "Design and natural science research on information technology." Decision support systems 15, no. 4 (1995): 251-266.

[31] Mystakidis, Stylianos, Niki Lambropoulos, Habib M. Fardoun, and Daniyal M. Alghazzawi. "Playful blended digital storytelling in 3D immersive elearning environments: a cost effective early literacy motivation method." In Proceedings of the 2014 workshop on interaction design in educational environments, pp. 97101. 2014.

[32] Nash, Susan Smith, and Michelle Moore. Moodle course design best practices. Birmingham: Packt Publishing, 2014.

[33] Nunamaker Jr, Jay F., Minder Chen, and Titus DM Purdin. "Systems development in information systems research." Journal of management information systems 7, no. 3 (1990): 89-106.

[34] Parmar, Dhaval, Jeffrey Bertrand, Sabarish V. Babu, Kapil Madathil, Melissa Zelaya, Tianwei Wang, John Wagner, Anand K. Gramopadhye, and Kristin Frady. "A comparative evaluation of viewing metaphors on psychophysical skills education in an interactive virtual environment." Virtual Reality 20, no. 3 (2016): 141-157.

[35] Peffers, Ken, Tuure Tuunanen, Marcus A. Rothenberger, and Samir Chatterjee. "A design science research methodology for information systems research." Journal of management information systems 24 , no. 3 (2007): 45 77.

[36] Pollard, Kimberly A., Ashley H. Oiknine, Benjamin T. Files, Anne M. Sinatra, Debbie Patton, Mark Ericson, Jerald Thomas, and Peter Khooshabeh. "Level of immersion affects spatial learning in virtual environments: results of a three-condition within-subjects study with long intersession intervals." Virtual Reality 24, no. 4 (2020).

[37] Rai, Arun. "Editor's comments: Diversity of design science research." MIS quarterly 41, no. 1 (2017): iii-xviii.

[38] Shapiro, Heather B., Clara H. Lee, Noelle E. Wyman Roth, Kun Li, Mine Çetinkaya-Rundel, and Dorian A. Canelas. "Understanding the massive open online course (MOOC) student experience: An examination of attitudes, motivations, and barriers." Computers \& Education 110 (2017): 35-50.

[39] Simon, H. A. "The Sciences of Artificial, Cambridge MA and London." (1996).

[40] Sportillo, Daniele, Alexis Paljic, and Luciano Ojeda. "Get ready for automated driving using virtual reality." Accident Analysis \& Prevention 118 (2018): pp. 102-113.

[41] Surprenant, Carol F., and Michael R. Solomon. "Predictability and personalization in the service encounter." Journal of marketing 51.2 (1987): pp. 86-96.

[42] Unreal Engine. Accessed June 2021.https://www.unrealengine.com/en-US/

[43] Van Seters, J. R., M. A. Ossevoort, J. Tramper, and Martin J. Goedhart. "The influence of student characteristics on the use of adaptive e-learning material." Computers \& Education 58, no. 3 (2012): 942-952.

[44] Vom Brocke, Jan, Robert Winter, Alan Hevner, and Alexander Maedche. "Special Issue EditorialAccumulation and Evolution of Design Knowledge in Design Science Research: A Journey Through Time and Space." Journal of the Association for Information Systems 21, no. 3 (2020): 9.

[45] Yoshinov, Radoslav Dakov, and Oleg Petrov Iliev. "The structural way for binding a learning material with personal preferences of learners." Труды СПИИРАН 60.0 (2018): pp. 189-215 